\title{
Reduction of epistemic uncertainty of a crash box model - experimental and numerical investigations
}

\author{
P. Wellkamp ${ }^{a^{*}}$ (iD \\ M. Meywerk \\ a Department for CAE methods at Volkswagen AG, Germany. Email: wellkamp.philipp@gmx.de \\ ${ }^{b}$ Department of Mechanical Engineering, Helmut-Schmidt-University, University of the Federal Armed Forces Hamburg, Germany. \\ Email: martin.meywerk@hsu-hh.de \\ ${ }^{*}$ Corresponding Author
}

http://dx.doi.org/10.1590/1679-78255370

\begin{abstract}
The high prediction accuracy of FEM calculations is an important prerequisite for their growing use in the field of crash load cases for vehicle body design. Although the simulation accuracy of crash calculations for steel structures already is at a high standard, continuous improvements and a further development of the simulation models are crucial in the field of vehicle design. Existing studies on the simulation accuracy for steel structures under crash load focus on the deformation behaviour of idealized component-like structures. These studies do not allow for conclusions on the simulation accuracy of crash calculations based on vehiclerelated components with increased geometric complexity as well as with different material combinations, joining techniques and bead structures. This was the reason for conducting the experimental and numerical investigations on crash boxes outlined in this paper. The goal was to investigate whether the FE code is capable of increasing the accuracy of the simulation of the experimental results by considering the information obtained from technical measurements.
\end{abstract}

\section{Keywords}

validation, uncertainty quantification, finite-element, energy absorption, automotive, simulation

\section{INTRODUCTION}

The realistic simulation of the deformation of steel structures is a prerequisite for their application in the vehicle body design. Numerous investigations focus on verifying numerical calculations for high-strength steel during impact scenarios in corresponding experiments. They verify simulations using idealized examples such as top hat profiles, square profiles or other thin-wall steel profiles of this type.

In Huh und Kang (2002), Oscar und Eduardo (2008) and Tarigopula et al. (2006) experiments with simulations for high-strength steel structures under axial load in the quasi-static and dynamic range are compared. This involves the investigation of different effects resulting from geometric details, impactor speed and other influencing factors. The investigations showed a high degree of correspondence between the experiment and simulation in terms of the deformation behaviour and the force-displacement characteristics so that the validated model allows for analysing additional effects on the energy absorption.

These results are also supported by the studies in Peixinho et al. (2003), Jones (1993) and Otubushin (1998). In Abedrabbo et al. (2009) the crash behaviour of high-strength steel tubes under axial load using FEM is analysed. The comparison of the results from the experiment with the simulation results showed that the FEM calculation offers high simulation accuracy, with regard to the differences in the force-displacement curves which result from effects during the deformation process. 
Yamashita et al. (2003) conducted a numerical study on the deformation behaviour under axial load of different top hat profiles made from different steel alloys. The authors analysed the buckling behaviour of a top hat profile depending on the weight, on different complex shapes of the cross-section and on imperfections. The numerical calculations were compared with data from the experiments. The results show high simulation accuracy for the force path, in particular for profiles made from high-strength steel. Reddy et al. [2015] also compared results from theory, simulation and experiments based on different cross-sections under axial load. The study showed a high degree of correspondence in the force-displacement characteristics and buckling behaviour so that the validated model allows for additional conclusive studies on the energy absorption of profiles with various cross-sections.

Further papers investigate the influence of parameter variations on the simulation results and compare it to the experimental results. In Fyllingen et al. (2008a) top hat profiles made from dual-phase steel under axial compression load using simulations and experiments are analysed. The investigation considers measured geometry data, variations in wall thickness and variations in material characteristics. The simulations are based on experiments which were conducted in Fyllingen et al. (2008b). The study analysed the influence based on a component characterization. It showed that the effects were most significant when the measured geometric data had been considered. In addition, the variations in wall thickness and in material characteristics also have considerable influence on the results when compared to the nominal model. The majority of the effects which result from the deviations in the component characteristics and which could be observed in the experiments were also shown in the simulations.

The significant influence on the simulation results which arises from considering the initial imperfections of the specimen during impact load and the resulting improvement in the simulation accuracy are confirmed by other papers. Xue et al. (2013) found, for example, that the dynamic progressive buckling of square tubes under axial load is sensitive to geometric imperfections. The calculation results indicate that the variations in the wall thickness have only a limited influence on the deformation behaviour whereas the geometrical deviations in the faces of the square tubes have significant influence on the deformation behaviour. These findings are confirmed by Eichmueller and Meywerk (2012), who also analysed the influence of the geometry on the buckling of square tubes under compression load. Their paper also states that appropriate FEM models show a high simulation accuracy when compared with real experimental results and when variations in the results of repeated experiments are considered. The study of Wellkamp et al. (2014) shows that this method can also be applied for crash boxes with a complex geometry and with fast load cases. The analysis of complex structures presented in this paper is based on these results.

The work presented by Kokkula et al. (2006) also relates to the model validation for complex structures. There, a system consisting of a cross member and longitudinal member made from aluminum was exposed to an impact with a coverage of the barrier of $40 \%$ and was analysed using both simulation and tests. A comparison of the force-time curves and deformation behaviour showed that there are still improvements required in terms of the degree of correspondence. The average force characteristics correspond well with the results from the tests.

The investigations of Xu and Wang (2016) and Xu et al. (2016) also cover steel structures with a complex geometry. $\mathrm{Xu}$ und Wang (2016) analyses the deformation behaviour of thin-walled top hat profiles with different tailor-welded blanks made from high-strength steel under dynamic axial load, both experimentally and numerically. The accuracy of the simulation model proved to be appropriate, so that the analysis of the material characteristics and thickness of the individual segments for the assessment of the crash behaviour of the top hat profiles and the results of the numerical calculations were used in the course of the study.

In Xu et al. (2016) the crash behaviour of an energy-absorbing structure consisting of square profiles and cross beams for subway vehicles made from steel is analysed. For this, the simulation results have been verified against the results from the physical trolley tests. It showed a high degree of correspondence between the test results and the simulation results so that the validated model can be used for further numerical parameter studies and structural optimizations.

Many of the referred papers cover the model validation of idealized thin-wall steel structures such as square tubes or top hat profiles. The degree of correspondence between the results from the numerical calculations and the experiments is already at a high level for these structures with regard to the deformation behaviour and characteristic curves. However, the model validation of component-like steel structures with complex geometry still requires further development. This refers to vehicle-related structures which consist of several individual parts and which comprise structural elements such as beads and holes as well as various joining techniques and material combinations. These structures form the basis for the experimental and numerical analyses in this paper. Initially, the simulation accuracy for the real deformation behaviour of such structures was determined using the FE code PamCrash. Then, areas for the improvement of the simulation results were determined. For this, a technical component and material characterization was performed by means of technical measurements. The measurement data enables the reduction of uncertainty 
concerning the component characteristics of the crash box within the boundaries set by the measurement tolerances. For this, the measured punch trajectory, experiment-specific material properties as well as geometry data from the photogrammetric measurement and gauge measurement were analysed, firstly under quasi-static load and then, under dynamic load. After that, the respective model was validated as a sub-model of a component system.

\section{Validation, verification and uncertainty quantification (VV \& UQ)}

Validation and verification are considered to be a process for increasing the reliability of a model and are not intended to prove its absolute accuracy (Robinson, 1997). The term "verification" refers to the process of verifying the accuracy of numerical mathematical solutions in the FE code, i.e. the correct creation of the model (Davis, 1992). In contrast, the term "validation" refers to the process of verifying the accuracy of the model with regard to the objective of the application, i.e. the creation of the correct model (Carson, 1986). Validating the correspondence of the simulation results with the results of the real experiments is referred to as "model validation". This involves the assessment of the reliability and credibility of the simulation model based on the simulation accuracy when compared with the real behaviour. In this context, the experiments must be configured in such a way that the validation domain covers the application domain entirely to avoid extrapolations. The calibration is a part of the validation. Model parameters are adapted in this iterative process.

The assessment of the simulation accuracy of a model and the related uncertainty analysis is an important prerequisite for creating reliable calculative predictions. The term "prognostic capability" refers to the ability to provide a reasonable and realistic simulation of crash and impact scenarios. Only if a numerical simulation can predict the deformation of a component under a certain load and whether the component will fail, can the simulation be appropriate. The prognostic capability of a numerical simulation also involves the approximately exact prediction of the real physical processes. The prediction can only be approximately precise and arbitrarily close to the reality. This is due to the character of numerics themselves. In order to properly assess the quality of a numerical solution, the design engineer needs to know the degree of approximation and the uncertainty. Hiermaier (2003) classifies the approximations and uncertainties into three levels: 1) Idealization of the structural geometry and of the application of the load; 2) Formulation of descriptive equations; and 3) Discretization of equations.

The analysis of the causes and consequences of uncertainties in the FE model is essential for the validation and for establishing trust into the experiment and simulation. Since, the analysis of uncertainties with regard to the validation is a major aspect in this study, the findings from the literature concerning this topic shall be summarized in this chapter. For this, the basic studies of Hanson and Hemez (2003), Hemez (2004), Hemez et. al. (2007), and Wojtkiewicz et. al. (2001) are referred to.

Before covering the prerequisites for reliable simulation models, the related terms shall be explained briefly in this section. In the relevant literature the term "uncertainty quantification" (UQ) is used. In this context, the term variability is often used as well. Gottschalk-Mazouz (2003) describes this term as the variation of a quantity over different elements of a class (e.g. the wall thickness of square tubes in a series of experiments). In contrast, uncertainty exists as to the quantity of a certain element in this class (e.g. the measurement accuracy for measuring the wall thickness distribution of a square tube). According to Booker (2004), an uncertainty analysis is the process of characterizing, assessing and analysing the different types of uncertainty (and variability) for complex decision problems. For complex computer models and physical models the term refers mainly to uncertainties in measurements, calculations, input parameters and modeling. The uncertainty analysis is an evaluation and assessment process.

The validation establishes the credibility of the simulation model. Hemez (2004) states that the credibility of simulation results shall comprise three major components: 1) the credibility of the prediction of experimental results; 2 ) the robustness of the prediction against fluctuations in the input parameters, uncertainty and missing information on the component; and 3) the prediction accuracy of models for which no measurement data is available. Wojtkiewicz et al. (2001) add that for numerical calculations there are both uncertainties about the physical characteristics of the analysed object and about the boundary conditions of the environment. It is widely accepted that for such systems the results from deterministic analyses are not sufficient to describe the response behaviour of the system appropriately and completely. Therefore, appropriate methods such as stochastic analyses must be applied to analyse non-deterministic effects as is the case in the work of Marczyk (1997) for various CAE disciplines or technical application scenarios.

Real physical systems are subject to both systematic and random deviations from an idealized system. There are various potential random deviations such as variations in geometry (imperfections due to manufacturing procedures), in material characteristics (elastic modulus and stress-strain curves) as well as in the boundary conditions and initial conditions (ambient temperature and impactor speed). Thus, in reality there are always differences between the 
particular systems. This means that the analysed objects and their environment never have the exact same characteristics. Refer to Alvin et al. (1996) for a more detailed description of potential sources for uncertainties and faults in computer models. These potential sources for uncertainties in predictive simulations are categorized as follows by Hanson and Hemez (2003):

- Uncertainty in mathematical models

- Uncertainty in material models

- Numerical variance

- Uncertainty in the initial conditions and boundary conditions

According to Frey and Rhodes (1999), Wojtkiewicz et. al. (2001) and laccarino (2009) uncertainty results from insufficient information on the actual value of a quantity or on the actual distribution of a variability. Within this context, the literature differentiates between the aleatoric uncertainty ${ }^{1}$, which is considered to be inherent and non-reducible, and the epistemic uncertainty ${ }^{2}$, which results from incomplete information and can be reduced (Trucano, 2000).

Statistical distribution functions can be used to consider these in the simulation model. The first step in the uncertainty analysis is to characterize the uncertainties in the system parameters. This includes both types of uncertainties whereas in most cases they cannot be clearly distinguished. The next step is to transfer the uncertainties to more complex simulation models. According to Hanson and Hemez (2003) the traditional use of statistical distribution functions for considering the variability of parameters is the only proven and reasonable method for considering uncertainties in the model.

The research question in this study is whether the information on the analysed object obtained from technical measurements, i.e. the reduction of the epistemic uncertainty, helps to increase the accuracy of simulations and which aspects are crucial for such information.

\section{Experiments}

This section gives a description of the experiment specimen as well as of the configuration and results of the quasistatic and dynamic experiments.

\subsection{Analysed object}

The analysed object is a crash box - shown in Figure 1 (left) - which consists of five individual parts: bulkhead plate, inside plate, outside plate, separate bracket and piece of crossmember. It is a vehicle-related steel structure similar to a crash box which was designed and manufactured with defined structural elements. The crash box measures approx. 200 $\mathrm{mm}$ in height, $100 \mathrm{~mm}$ in width and $78 \mathrm{~mm}$ in depth. There are beads opposite each other in the center of the sides as well as in the front area on the top and bottom of the experiment specimen. This bead structure shall cause a defined deformation and energy absorption of the crash box during impact. The bracket is part of the crash management system and is separated below the edge of the piece of crossmember for the experiments. The inside and outside plates are cold-formed U-shaped profiles which are spot-welded in the area of the overlaps. The inside and outside plates are the load-bearing components of this experiment specimen, and the load path runs through them during impact. They are made from dual-phase steel with a nominal yield strength of $780 \mathrm{MPa}$.
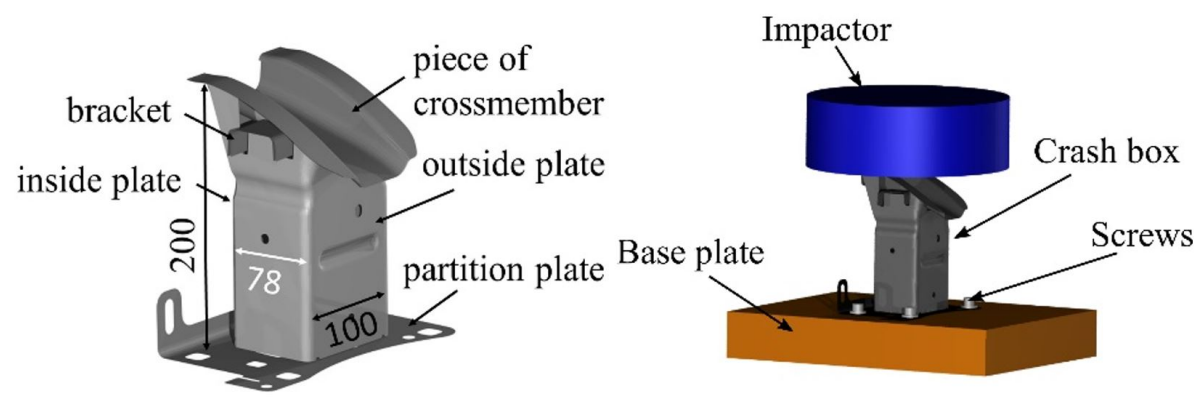

Figure 1: Individual parts of the crash box (left) and Simulation model (right).

\footnotetext{
${ }^{1}$ From "alea", Latin for "dice"

${ }^{2}$ From "epistéme", Greek "knowledge"
} 


\subsection{Experimental configuration}

\subsubsection{Configuration for quasi-static experiments}

Figure 2 shows the experiment configuration for the quasi-static compression load experiment on a servo-hydraulic universal testing machine. In the first series of experiments the punch has an inclination of 0-degrees. In the second series of experiments the punch has an inclination of 10-degrees. The partition plate which is welded to the crash box is bolted to the base plate using four screws. In the following sections the different series of experiments are called 0 degree experiments and 10-degree experiments.

The base plate moves upwards along the guides with a speed of $30 \mathrm{~mm}$ per minute. The experiment specimen is compressed in axial direction between the base plate and the stationary punch. For each series of experiments, i.e. for both load angles, five valid experiments are performed and evaluated. The force in direction of the compression load is measured using a load cell which is located above the punch.

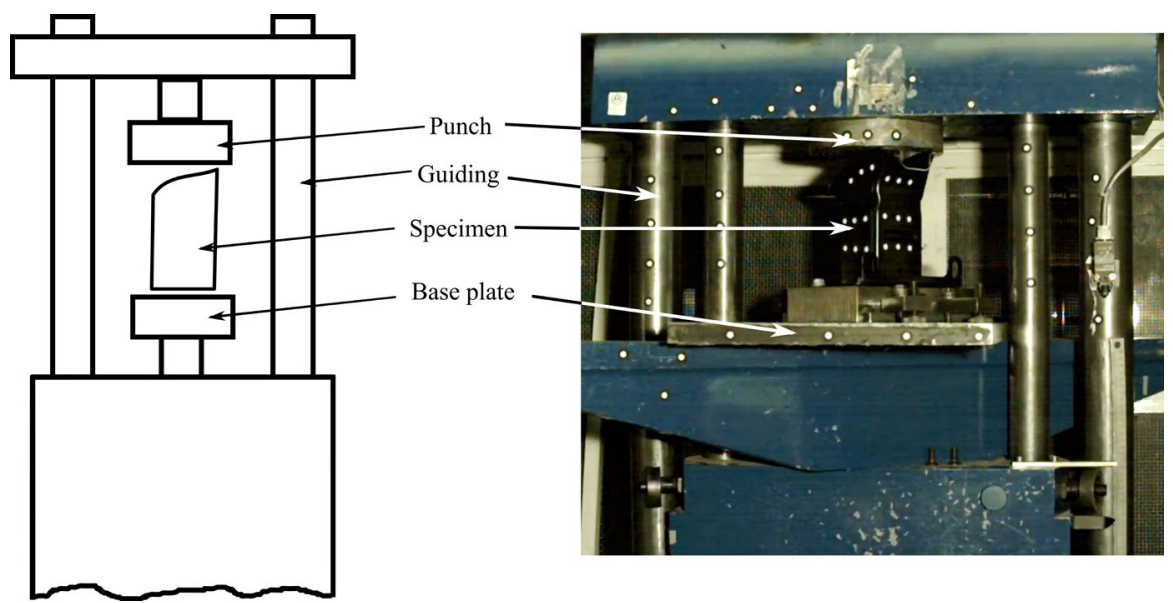

Figure 2: Left: schematic view of the universal testing machine; Right: experiment stand of the universal experimenting machine

\subsubsection{Configuration for dynamic experiments}

The experiment configuration for the dynamic impact experiments is shown in Figure 3. The energy is introduced into the crash box via the piece of crossmember by the impactor which is secured to a laterally and vertically guided sled with a total weight of approx. $300 \mathrm{~kg}$. The partition plate of the profile to be analysed is mounted centrally to the base plate and the load is introduced centrally via the impactor on the sled. The force signals are recorded by the piezoelectric load cell with a measurement frequency of $10 \mathrm{kHz}$ which is located behind the base plate. A CFC 600 signal filter is used. As for the initial conditions and boundary conditions, the experiments were performed successively at room temperature.
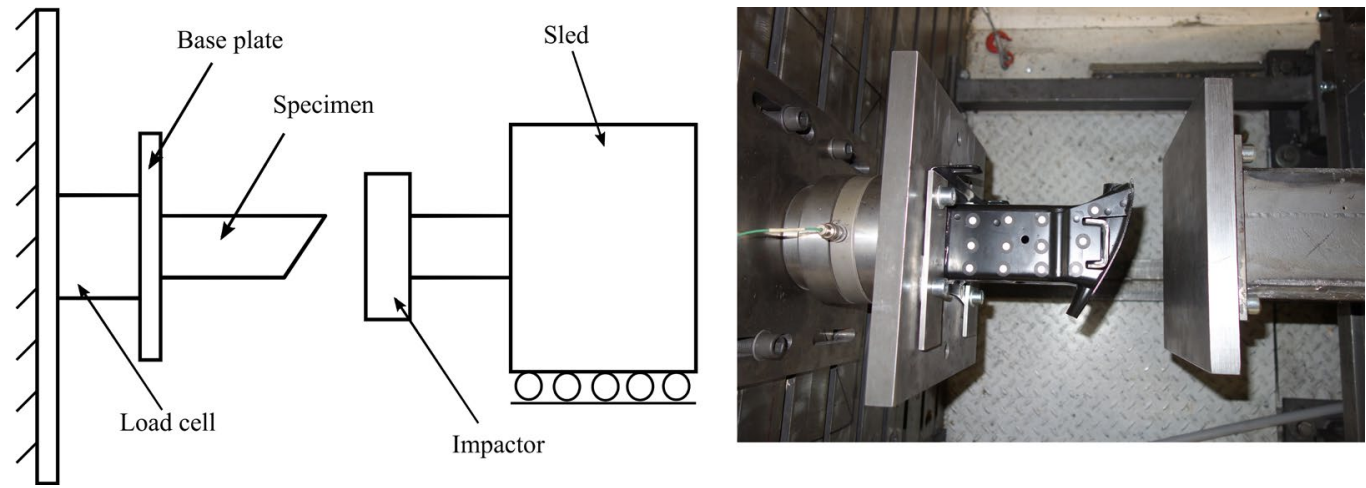

Figure 3: Schematic view of the universal testing machine (left); Experiment stand (right) 


\subsection{Experimental results}

\subsubsection{Quasi-static experiments}

The experimental results of the two series of experiments are described by means of the force-displacement curves. Figure 4 shows the curves for the 0-degree-experiments on the left. The high degree of correspondence of the forcedisplacement characteristics indicates a good reproducibility of the experiments. Figure 4 shows the force-displacement characteristics for the 10-degree experiments on the right. This series of experiments also shows a high reproducibility of the experiments as well.

When compared to the other experiments, experiment 8 shows a slight deviation of the force curve after approx. $38 \mathrm{~mm}$. Generally, the curves show a high degree of correspondence with regard to the shape and level of the curve up to a punch travel of approx. $65 \mathrm{~mm}$ or up to the fourth rise. After that, slight deviations can be observed. When the force is introduced with an inclination of 10-degrees, the crash box is deformed in a different way towards the outside so that the force characteristics deviate from each other as of a punch displacement of $60 \mathrm{~mm}$.
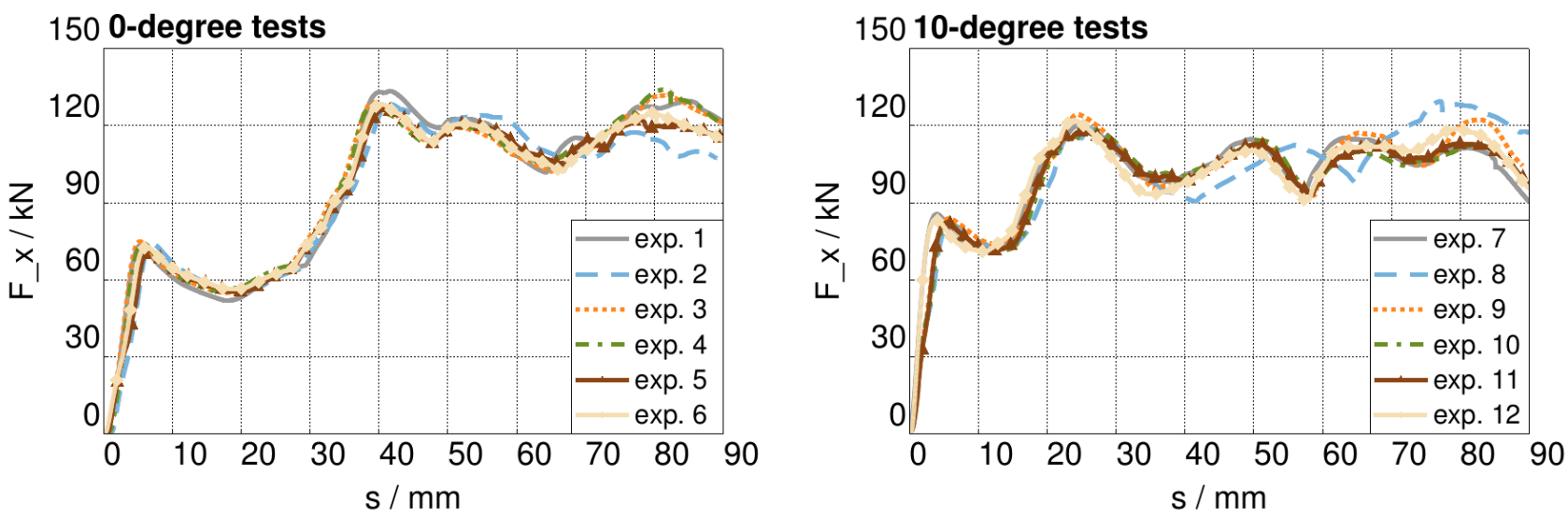

Figure 4: Force-displacement-curves of the quasi-static experiments.

\subsubsection{Dynamic experiments}

Two dynamic load cases with different load angles were analysed. The first series of experiments comprised five impact experiments with a load angle of 0-degrees. For the second series of experiments the load was introduced into the structure at an angle of 10-degrees. In the following section these load cases are referred to as 0-degree experiments and 10-degree experiments as well.

Refer to the tables 1 and 2 for the measured impactor speed during impact. The average value is indicated with $\bar{x}$, the kinetic energy is defined as $E_{\text {kin }}$ and $\mathrm{F}$ stands for the force. The reproducibility of the experiments was high with an impact speed variance $R$ of $1.2 \%$ for the 0 -degree experiments and $1.6 \%$ for the 10 -degree experiments.

Table 1: Results of dynamic 0-degree experiments.

\begin{tabular}{ccccccccc}
\hline Experiment & $\mathbf{0 1}$ & $\mathbf{0 2}$ & $\mathbf{0 3}$ & $\mathbf{0 4}$ & $\mathbf{0 5}$ & $\overline{\boldsymbol{x}}$ & $\mathbf{R}$ & $\mathbf{R} /[\%]$ \\
\hline Impactor-speed $[\mathrm{m} / \mathrm{s}]$ & 8.26 & 8.26 & 8.33 & 8.16 & 8.23 & 8.25 & 0.37 & 1.2 \\
$\mathrm{E}_{\text {kin }}[\mathrm{J}]$ & 10279 & 10279 & 10451 & 10029 & 10195 & 10247 & 422 & 4.1 \\
Deformation $[\mathrm{mm}]$ & 98 & 100 & 102 & 101 & 102 & 100 & 4 & 4.0 \\
$\mathrm{~F}_{\text {average }}[\mathrm{kN}]$ & 99 & 99 & 93 & 98 & 94 & $97 \pm 2.8$ & 6 & 6.4 \\
$\mathrm{~F}_{\max }[\mathrm{kN}]$ & 167 & 177 & 180 & 176 & 193 & $179 \pm 8,4$ & 26 & 9.0 \\
\hline
\end{tabular}


Table 2: Results of dynamic 10-degree experiments.

\begin{tabular}{ccccccccc}
\hline Experiment & $\mathbf{0 6}$ & $\mathbf{0 7}$ & $\mathbf{0 8}$ & $\mathbf{0 9}$ & $\mathbf{1 0}$ & $\overline{\boldsymbol{x}}$ & $\mathbf{R}$ & $\mathbf{R} /[\%]$ \\
\hline Impactor-speed $[\mathrm{m} / \mathrm{s}]$ & 8.33 & 8.23 & 8.23 & 8.20 & 8.24 & 8.24 & 0.37 & 1.6 \\
$\mathrm{E}_{\text {kin }}[\mathrm{J}]$ & 10451 & 10195 & 10195 & 10195 & 10365 & 10229 & 339 & 3.2 \\
Deformation $[\mathrm{mm}]$ & 90 & 87 & 92 & 86 & 88 & 88 & 6 & 6,8 \\
$\mathrm{~F}_{\text {average }}[\mathrm{kN}]$ & 108 & 113 & 103 & 114 & 111 & $110 \pm 3.9$ & 11 & 10,0 \\
$\mathrm{~F}_{\text {max }}[\mathrm{kN}]$ & 167 & 166 & $\mathbf{1 6 0}$ & $\mathbf{1 5 6}$ & $\mathbf{1 6 3}$ & $\mathbf{1 6 2 \pm 4 , 0}$ & $\mathbf{1 2}$ & 7.4 \\
\hline
\end{tabular}

When compared with the series of quasi-static experiments, the load level for this series of experiments was higher due to structural inertia effects. The force-time characteristics of the 0-degree-experiments in Figure 5 showed a high reproducibility. The force-time characteristics of the 10-degree-experiments showed a good reproducibility up to the fourth rise in the force curve at approx. $12 \mathrm{~ms}$. After that, the curves deviate from each other. When the load was introduced into the crash boxes at an angle, a bending load was exerted on the crash boxes. The friction between the impactor and crash box varies for each particular experiment, because the impactor surface is roughened before each experiment. This is necessary to better control the lateral bending load. However, the bending behaviour of the crash boxes at the end of the deformation deviates so that the load path in the crash boxes showed slight deviations as well. This resulted in a deviation of the force curves as of the fourth rise within the deformation period in which the lower buckle in the crash box was formed.
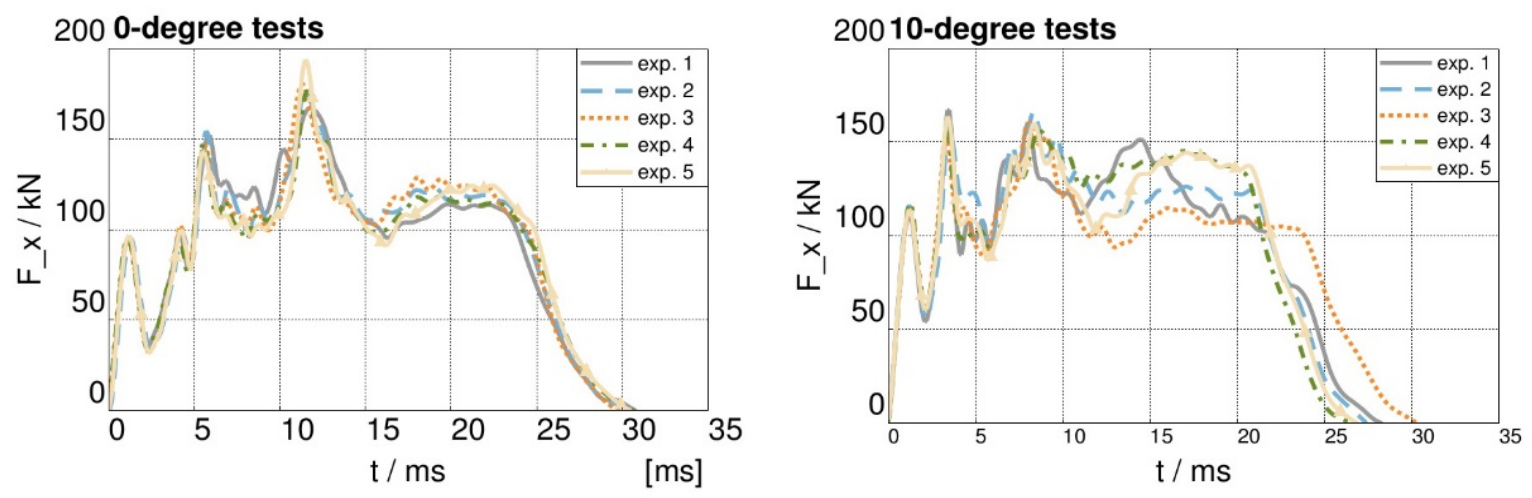

Figure 5: Force- time curves of the dynamic experiments.

\section{Simulation model}

The FE model of the crash box shown in Figure 1 (right) consists of shell elements with an average edge length of 2 $\mathrm{mm}$ for all parts. For the shell elements the Belytschko-Tsay formulation (Belytschko and Tsay, 1983) with five integration points along the thickness of the shell element and one integration point on the shell element plane was used. The contact thickness corresponds to the physical thickness of the individual parts. The effects caused by the forming process were considered.

The material model for the load-bearing inside and outside plates is the elastic-plastic material model MAT103 with strain rate dependency in PamCrash.

The constitutive equation of the material model implemented is an elasto-plastic model [ESI, 2017]. The strains are decomposed into elastic and plastic part

$\varepsilon=\varepsilon_{e}+\varepsilon_{p}$

and the yield condition is based on the von Mises equivalent stress

$\sigma_{v}=\sqrt{\sigma_{x}^{2}+\sigma_{y}^{2}-\sigma_{x} \sigma_{y}+3 \tau_{x y}^{2}}$ 
or the elasticity the linear elastic assumption

$\delta=E \varepsilon(3)$

is assumed. Concerning the hardening behaviour of the material the following stress-strain curves for different strain rates (Figure 6) are considered in the numerical calculations.

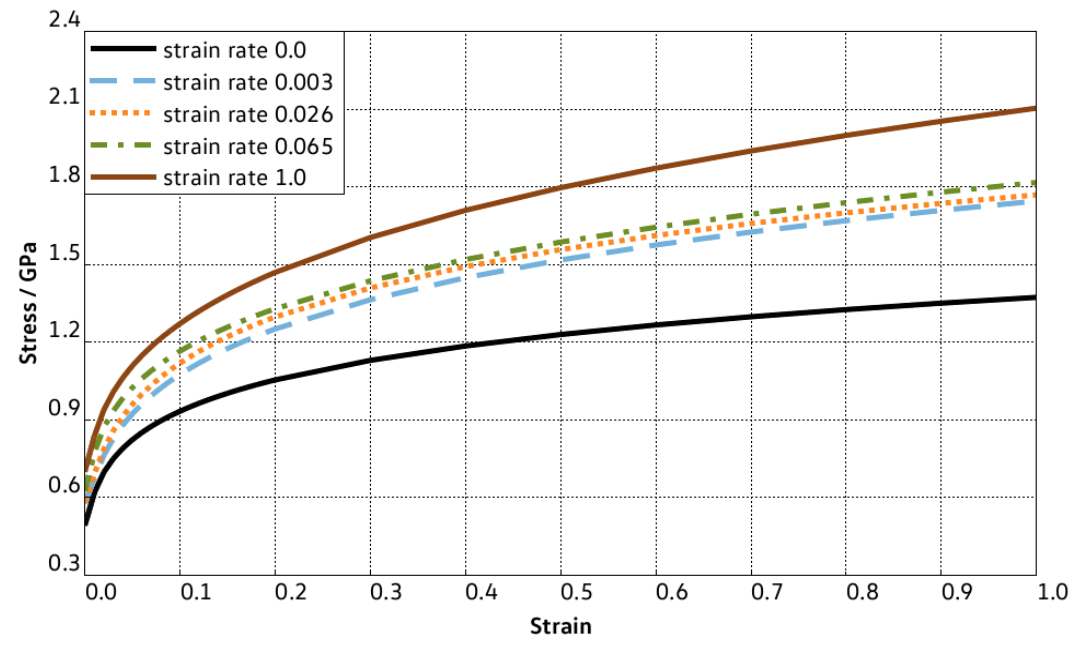

Figure 6: Strain-rate depended stress-strain curves.

To minimize the inertia effects the strain rate dependency of the stress-strain curve in the material card is scaled in accordance with the computing time. The punch and the base plate are modeled as rigid bodies. The base plate travels from the bottom upwards in direction of the compression load. The coefficient of friction between the contact surfaces is 0.3 . The surface of the base plate is roughened. Thus, the value of the coefficient of friction is slightly higher than the value of (Popov, 2009).

\section{Correspondence between experiment and simulation}

As stated in the theory section above the epistemic uncertainty is a potential lack of information on the experiment specimen and the boundary conditions of the experiment caused by missing information from technical measurements. This type of uncertainty can be reduced by proven methods such as the photogrammetric measurement of the geometry and wall thickness, the material characterization and the trajectory measurement. This chapter describes the influence of considering various data obtained from the measurements on the simulation results in the model.

The focus is on the force path in direction of the compression load and on the visual comparison of the deformation behaviour and the remaining length. The visual comparison of the deformation behaviour indirectly aids drawing conclusions on the accuracy of the simulation compared to the experiments with regard to the introduced momentum and lateral forces. The question here is whether the information obtained from the component and material characterization helps to increase the simulation accuracy significantly and which factors have the most influence. For this purpose the simulations with information of component characterization is compared with the nominal model. The characteristics of the nominal model is defined in table 3.

Table 3: Simulation nominal

\begin{tabular}{cc}
\hline Model criteria & Description \\
\hline Punch trajectory & Idealized linear z-trajectory \\
Material characteristics & Material database \\
& (see figure 6) \\
Geometry & CAD drawing \\
Thicknesses of plates & CAD drawing \\
Material model & Elastic-plastic \\
\hline
\end{tabular}




\subsection{Reduction of the epistemic uncertainty for validating the quasi-static load cases}

First, the simulation models are validated against the quasi-static experiments. Then, the model is validated for the dynamic load cases. This covers a wide range of load cases. These investigations are the basis for conclusions to be drawn on the influence of the information obtained from the component characterization on the simulation model.

\subsubsection{Punch trajectory}

The measurement of the punch trajectory is a characterization of the boundary conditions of the experiment and was performed during, instead of before the experiment, as was also the case for other aspects of the analysis as well which are described later in this chapter. The trajectory measurement was performed for two main reasons: First for comparing the movements of points on the experiment specimen and the impactor with those of the simulation. Second as an input parameter for controlling the impactor travel or trolley travel to assess the influence of the actual measured movement compared with the idealized movement.

Table 4: Characteristic variables of the force curve up to the first fall of the force curve.

\begin{tabular}{|c|c|c|}
\hline Curve & Max. Load [kN] & Initial Gradient [kN / mm] \\
\hline Experiment & 8.26 & 8.26 \\
\hline $\begin{array}{l}\text { Simulation } \\
\text { prescribed trajectory }\end{array}$ & 10279 & 10279 \\
\hline $\begin{array}{c}\text { Simulation } \\
\text { nominal }\end{array}$ & 98 & 100 \\
\hline
\end{tabular}

According to the overall force curve in Figure 7 the influence of the measured trajectory compared to the initial model with an idealized punch travel is low. For the comparison the respective experiment has been used for which the implemented trajectory has actually been measured. The main difference is the initial rise and the load level during the first fall of the force curve. The almost linear initial gradient of the nominal model is too high when compared to the experiment, as is the force level during the first fall of the force curve. Both quantities are improved (see table 4). For the initial gradient the recorded travel in direction of the compression load and thus, at the same time, the resilience or stiffness of the machine are considered. To summarize, the elastic part and the force level could be improved by considering the measured punch trajectory.

\subsubsection{Material characteristics}

This section describes the influence of the material characteristics which were obtained from samples of the experiment specimens used in this series of experiments. The initial model comprises a material model with strain-ratedependent stress-strain curves of the analysed hardened dual-phase steel of the crash boxes. The maximum permissible tolerance range for the yield point of the analysed dual-phase steel specified by the manufacturer is $\pm 50 \mathrm{MPa}$. The experiment-specific stress-strain curves obtained from samples of the experiment specimens are compared with those of the existing material model. For this, tensile specimens were taken from a non-strain-hardened area of four components, and the stress-strain curves were determined using a single-axis tensile experiment.

When the force characteristics of the initial model are compared with the stress-strain curve from the existing material model and from the model which considers the experiment-specific stress-strain curves, there is a slight deviation as of the second force peak at a punch travel of approx. $40 \mathrm{~mm}$. Overall no significant difference can be observed.

The analysis shows that (i) there are only slight deviations in the material characteristics between the existing stressstrain curves from the material map and the stress-strain curves obtained from the material characterization performed as part of this study and that (ii) these slight deviations do not have a significant influence on the force curve of the compression load simulation (Figure 8).

\subsubsection{Thickness measurement}

The thickness of the plates of the crash boxes are made from is subject to process variations in manufacturing. In this section the effect of considering the measured thickness shall be described. For this, four photogrammetric measurements of the thickness distribution of the crash boxes are performed. The nominal thickness of the inside plate is $1.8 \mathrm{~mm}$ whereas the nominal thickness of the outside plate is $2.0 \mathrm{~mm}$. The four measurements showed a clear trend towards a higher thickness than indicated in the drawing. Three measurements showed an increase in thickness of 0.1 
$\mathrm{mm}$ for both the inside and outside plate when compared with the nominal value in the drawing. One measurement even showed a thickness of the internal plate of $2.2 \mathrm{~mm}$. The thickness for the inside plate is increased to $1.9 \mathrm{~mm}$ and to $2.1 \mathrm{~mm}$ for the outside plate. The effects caused by the forming process are calculated based on the increased thicknesses.

Considering the increased thickness values obtained from the measurement results in a higher force curve. Figure 9 shows a comparison with the results from the calculations where nominal input parameters were used. It can be observed that the increase of the high force peaks is disproportionate to the increase of the lower force peaks. However, the curve characteristics do not change significantly when this measure is applied. Zhang and Zhang (2016) and Abramowicz and Wierzbicki (1983) confirm this effect. To summarize, the estimation of the force is too high compared to the experimental results when the thickness is increased. The section below describes the effects of combining this measure with all influencing variables from other measurements.

\subsubsection{Geometry measurement}

In this study the focus is on the component geometry and manufacturing-related deviations from the drawing geometry. The geometry data of the crash box obtained from photogrammetric measurements on the component can be considered by means of morphing. For this, the existing grid of the initial model is projected onto the model geometry obtained from the measurements. When doing this, the shape of the elements and node IDs remain identical so that the simulation results from the initial model based on the drawing geometry can be compared directly with the simulation results from the new model based on the measurement data whereas the effects from different discretizations play only a minor role.

When comparing the force characteristics of Figure 10, significantly improved simulation accuracy can be observed due to the geometry data being considered. Especially the initial rise and the force level during the first fall in the force curve now show a high degree of correspondence with the experiment. The correspondence of the second rise in the force curve and the characteristics of the second force peak have also been improved significantly by this measure.

\subsection{Combination of the information obtained from measurements}

In the section above the effects resulting from considering influencing variables obtained from measurements, such as the punch trajectory, the stress-strain curve, the geometry and the thickness, were analysed independently from each other. This section describes effects of combining all four measures.

If all four aspects are considered in the simulation model there is a high degree of correspondence between the simulation curve and the experiment curve, and at the same time there are significant improvements compared to the initial model with nominal input parameters. The curves in Figure 11 show a high degree of correspondence with regard to the initial rise, the force level, the second rise in the force curve, the characteristics and the second force peak. However, the last rise of the force curve is not yet represented in the model where all four measurements are considered.

The significant improvement of the simulation accuracy using the simulation model which considers the information from the component characterization can also be observed in the deformation images (Figure 12). The representation of the buckles in the inside plate in the upper area of the crash box is more realistic. Also the characteristic buckling of the outside plate is more realistic in the improved model when compared with the experiment. Overall the deformation behaviour of the improved model corresponds far more with the deformation behaviour of the experiment specimen.

\subsection{Transfer to 10-degree load case}

When transferring the simulation model to the second load case, the punch is inclined by 10-degrees so that the load is introduced differently into the object to be analysed. A comparison of the curves in Figure 13 and of the images of the final deformation in Figure 14 shows that the model considering the information from the measurements shows a high simulation accuracy for the 10-degree load case as well.

According to Figure 13 the degree of correspondence of the curves is also improved when compared with the initial model which already corresponds well with the experiment curve up to the second force peak at a punch travel of approx. $25 \mathrm{~mm}$. The improvement relates primarily to the initial rise of the force curve, the force levels at the force peaks and the further course of the curve after the second force peak. After the third rise of the force curve or a punch travel of approx. $50 \mathrm{~mm}$ the force characteristics deviate from each other.

However, the variance as of this point can also be observed during the five experiments which were performed. The images of the final deformation show that the modified model also helps to better represent the characteristic buckling in the upper area of the crash box. The deformation images in Figure 14 also show a significant improvement with regard to representing the buckling in the upper area of the crash box when compared with the nominal initial model. 


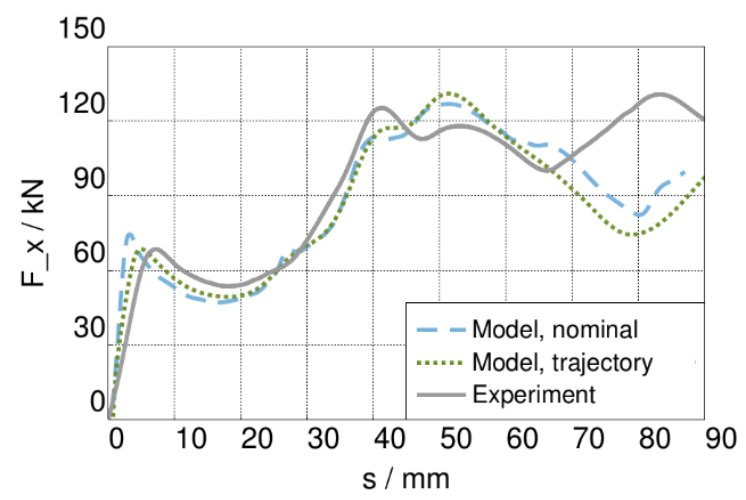

Figure 7: Forces: Punch trajectory; 0-dregree load case.

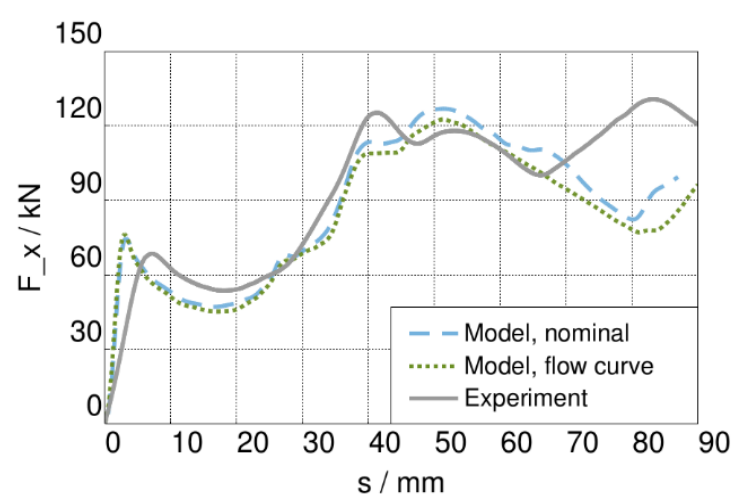

Figure 8: Forces: Stress-strain curve; 0-degree load case.

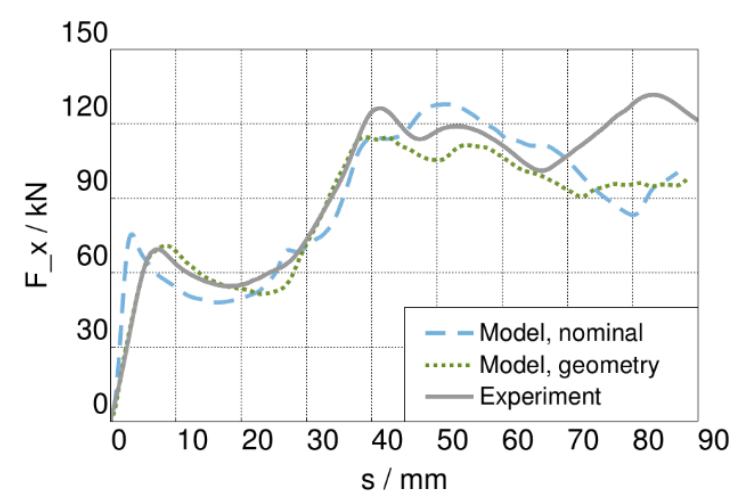

Figure 9: Forces: gauge measurement; 0-degree load case.

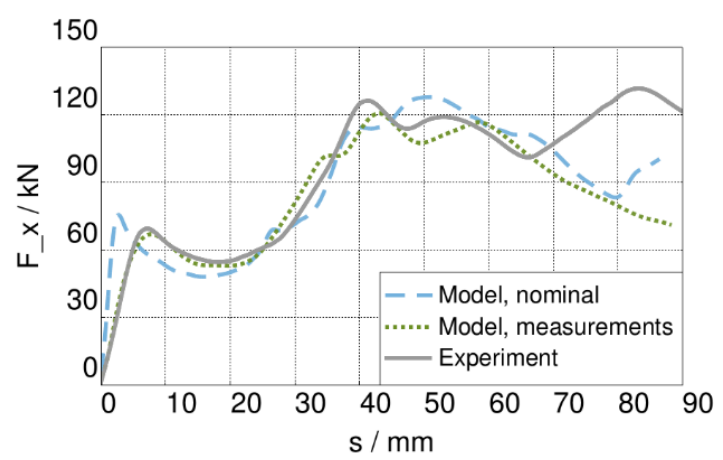

Figure 10: Forces: Geometry measurement; 0-dregree load case. 


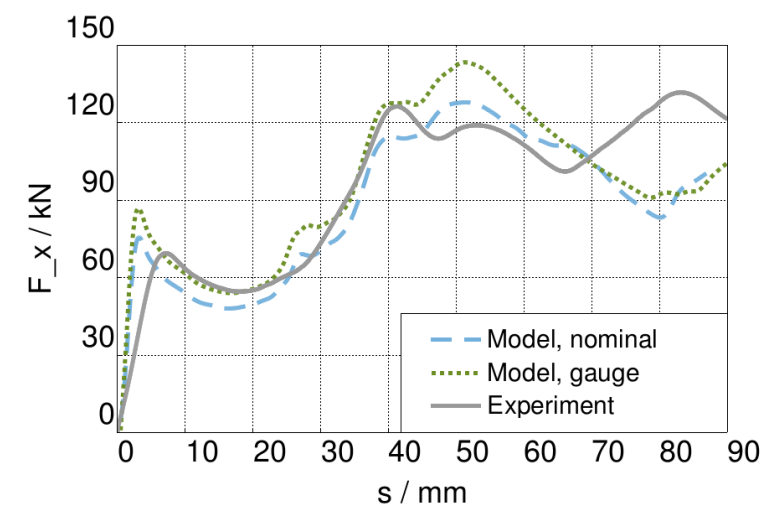

Figure 11: Forces: All measurements; 0-degree load case.

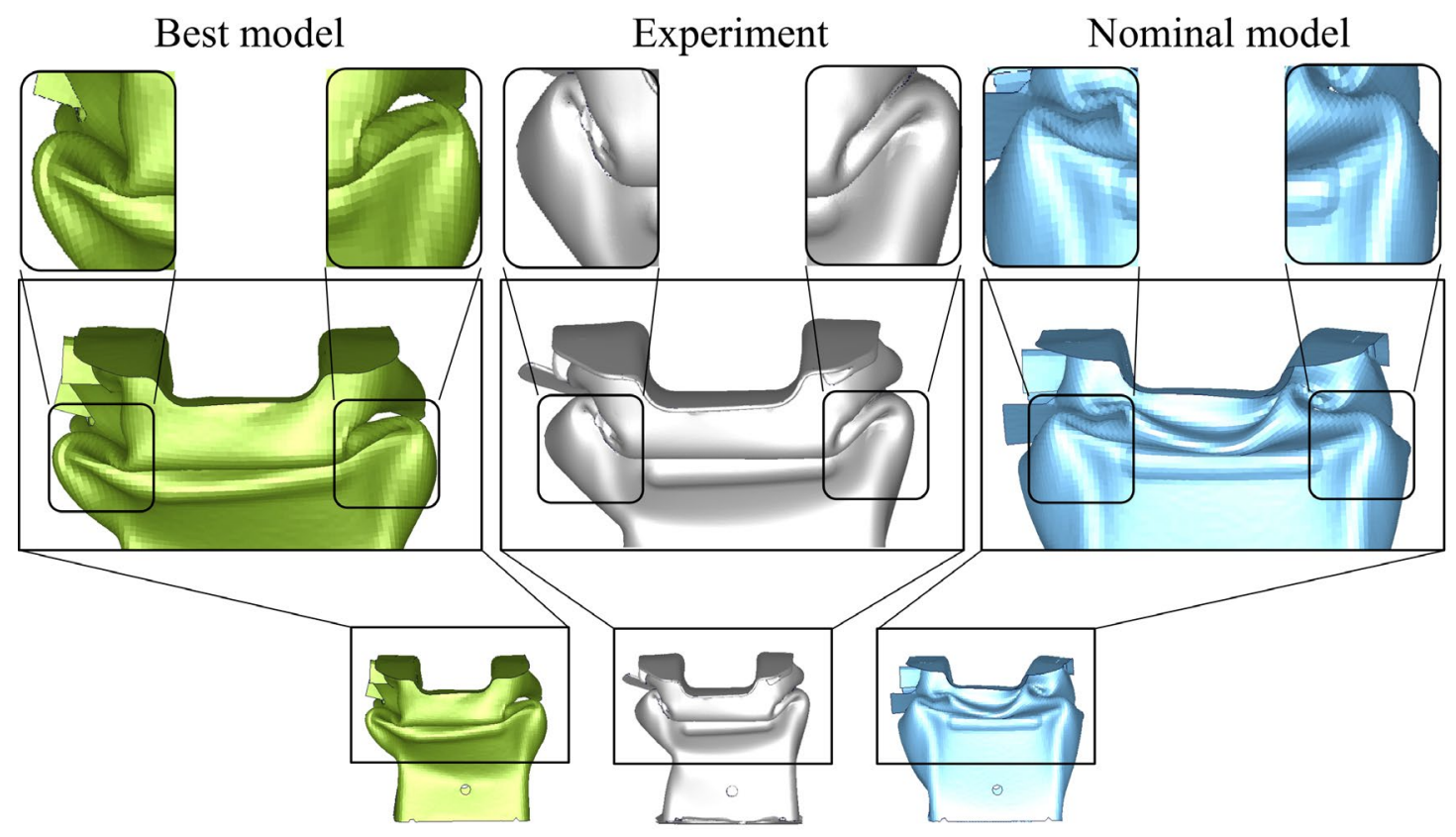

view outside plate

Figure 12: Comparison of the final deformation for the 0-degree load case. Left: best model with information from component measurements; Center: photogrammetric measurement of experiment specimen; Right: nominal model.

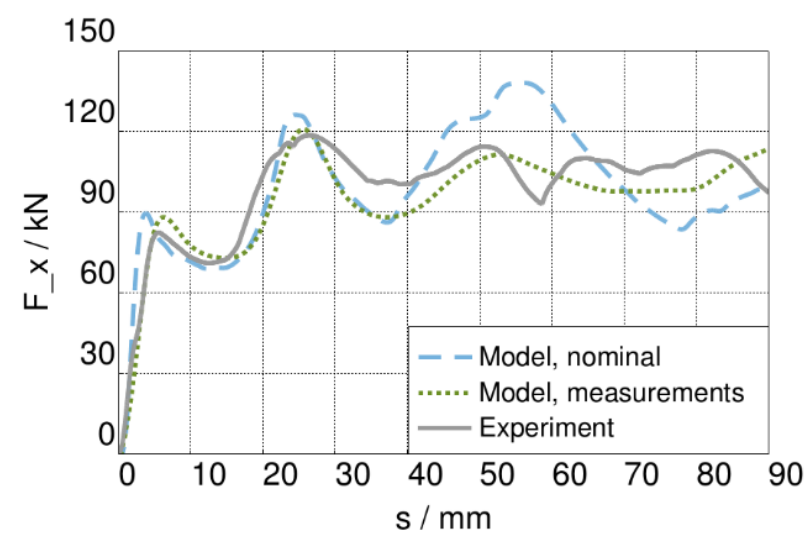

Figure 13: Forces: Consideration of all measurements; 10-degree load case. 
Best model

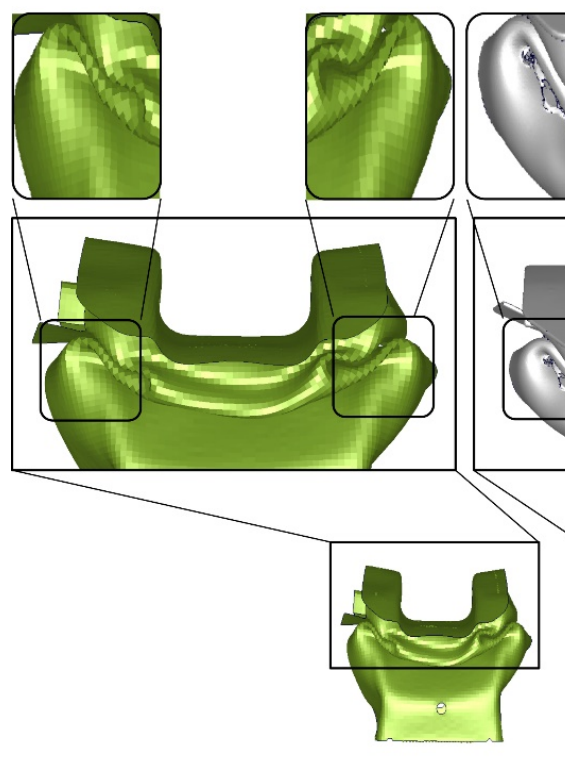

Experiment
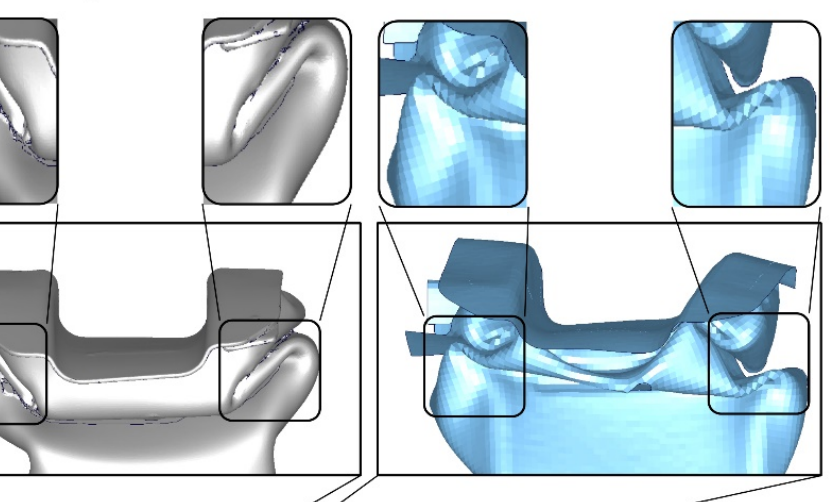

view inside plate

Figure 14: Comparison of the final deformation for the 10-degree load case. Left: best model with information from component measurements; Center: photogrammetric measurement of experiment specimen; Right: nominal model.

\subsection{Validation of the model with dynamic load cases}

After the model has been verified in the quasi-static field it is validated using dynamic load cases.

\subsubsection{0-degree load case}

The graph in Figure 15 shows a comparison of the force characteristics from the simulation which considers the measurement data, the nominal initial model and the experiment (average curve from five experiments). Up to the second fall of the force curve the force characteristics from the experiment and from the nominal initial model correspond well; after that, a high deviation can be observed. The improved model which considers the information from the component characterization shows a significant improvement in the correspondence of the curves. Here, the force characteristics correlate better with the experiment curve during the third buckling and at the end of the curve. According to the CORA evaluation (Thunert, 2012) the degree of correspondence increases from $82 \%$ to $95 \%$. Thus, the model can well be transferred to the fast load case.

To assess the degree of correspondence between the experiment and the simulation it is crucial to consider both the force characteristics and the correspondence of the deformation behaviour. Figure 17 shows sectional views of the crash box from the simulation and the experiment in the state of final deformation. On the left the contours of the model which considers measurement data (green) and the specimen (grey) are shown whereas on the right the initial model (blue) and the specimen (grey) are shown. First of all, the remaining length has been improved which is an important criterion. In addition, the characteristic buckling in the upper left area as well as on the left face are represented significantly better when compared with the initial model. Overall a significant improvement in the degree of correspondence of the experimental and numerical results with regard to the force curve-time curves and the deformation behaviour could be observed.

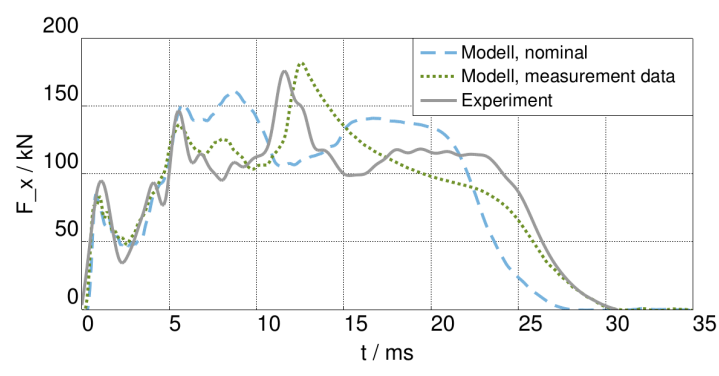

Figure 15: Forces: Dynamic 0-degree load case. 


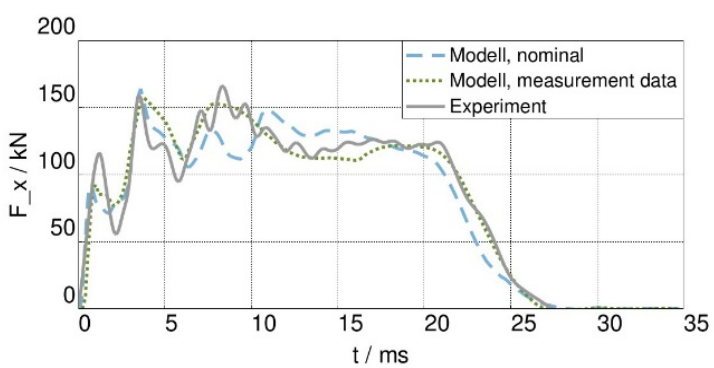

Figure 16: Forces: Dynamic 10-degree load case.

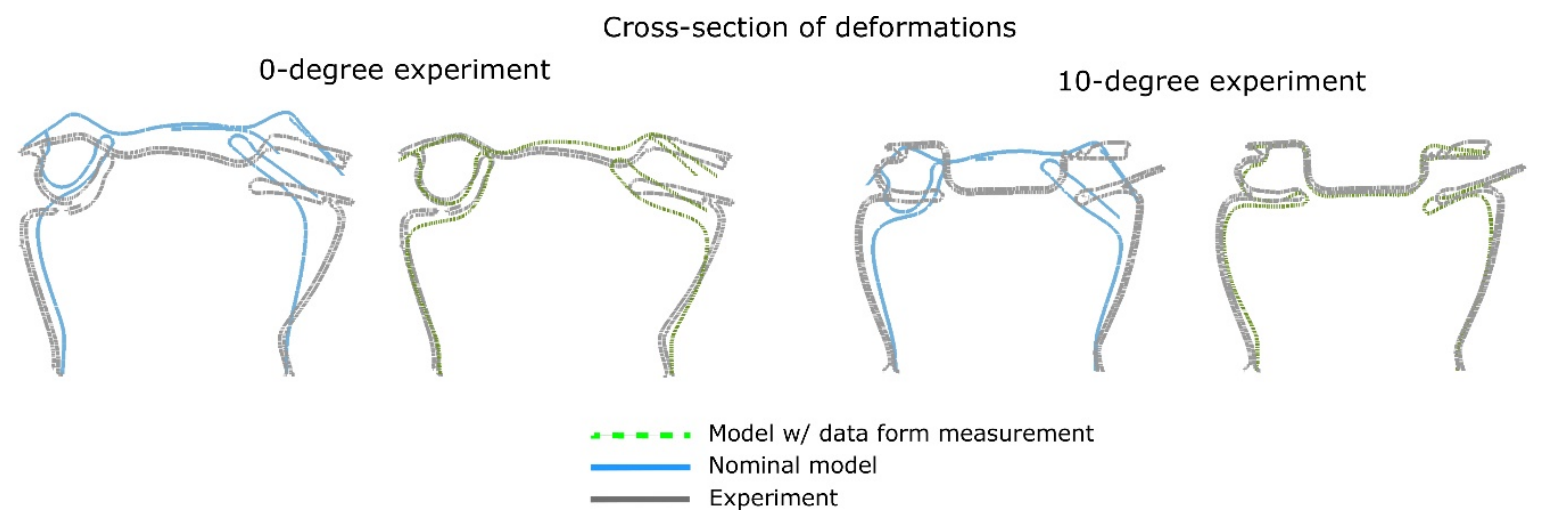

Figure 17: Sectional views of crash boxes in the state of final deformation. Left: 0-degree experiment and right: 0-degree experiment.

\subsubsection{0-degree load case}

As in the quasi-static field the simulation accuracy of the model is analysed both for the 0-degree load case and the 10-degree load case where the load is introduced at an angle to show that the optimizations are suitable for different load cases.

The graph in Figure 16 shows the force-time curves from the simulation which considers measurement data, the initial model and the experiments. Here, a high degree of correspondence between the curve of the initial model and the experiment curve can already be observed. There is a deviation at the third force peak. In this area, the model which considers measurement data shows an improved degree of correspondence between the experiment and the simulation. This also applies for the end of the curve so that the degree of correspondence could be improved slightly by 2 percentage points from $89 \%$ to $91 \%$ according to the CORA evaluation..

The sectional views of the crash boxes in the state of final deformation on the right in Figure 17 show a significant improvement in the deformation of the piece of crossmember, in the characteristic buckling on the top right of the compressed crash box as well as in the concavity of the faces on the left and right of the crash box. The contours of the deformed experiment specimen and the simulation which considers measurement data are almost congruent. Thus, the degree of correspondence could be improved slightly in terms of the force characteristics and significantly in terms of the deformation behaviour for the 10-degree experiments as well.

\section{Assessment of the validated model in a component scenario}

Having validated a high degree of correspondence on the component level, this section describes the crash box model as a sub-model of a model with higher complexity. The degree of suitability of the validation of an already validated sub-model in the validation hierarchy will be demonstrated. For this, longitudinal member systems are analysed numerically and experimentally. The degree of complexity with regard to the geometry, number of components and joining techniques increases with the increasing application relevance.

\subsection{Analysed object and Test configuration}

The longitudinal member system consists of a total of 19 individual parts. The most critical components for the energy absorption and the deformation behaviour are the crash boxes and longitudinal member made from DP780. 
The experiment configuration (Figure 18) consists mainly of the trolley, the experiment specimen, a reinforcement between the engine mountings and the sensor wall. The longitudinal member system which is secured to the trolley is driven straight against the wall with a coverage of $100 \%$ and a speed of $7.78 \pm 0.03 \mathrm{~m} / \mathrm{s}$. The test is performed three times to obtain authoritative results and to allow for conclusions on the reproducibility.

The model of the longitudinal member system consists of 2-mm-shell elements and includes the validated submodel of the crash box which contains the geometry information obtained from the component measurement.

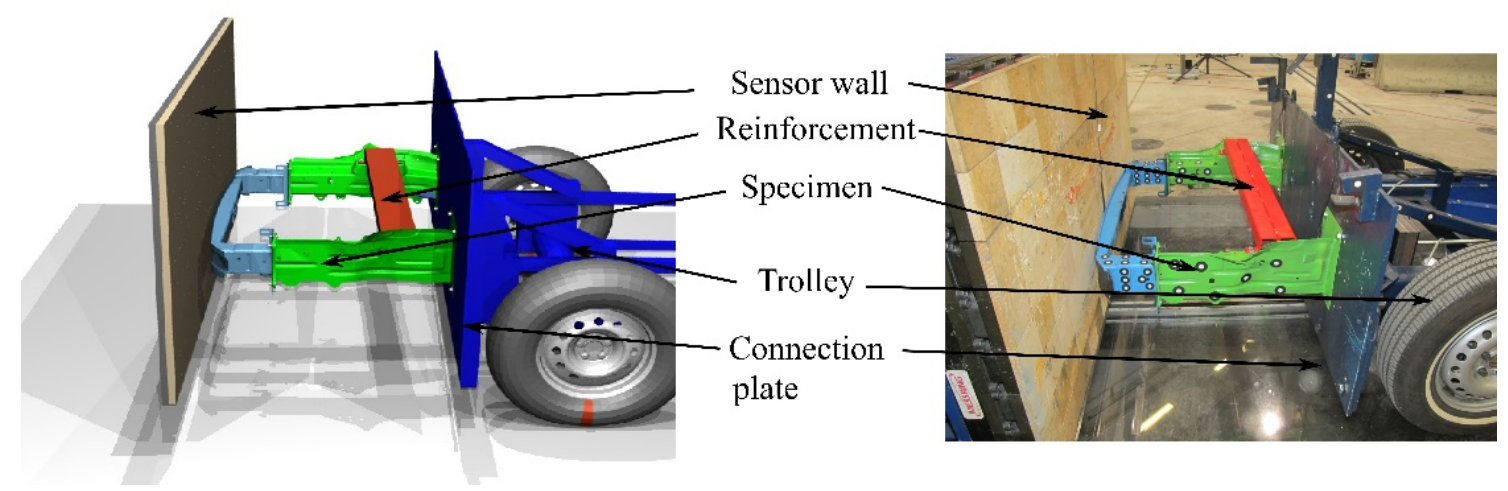

Figure 18: Left: Simulation model; Right: Test configuration.

\subsection{Degree of correspondence of the simulation with the Tests}

Figure 19 shows the force-time curves of the left and right longitudinal members. There is a sufficient degree of correspondence between the force characteristics of the simulation and the tests whereas the experiment curves show a good reproducibility amongst each other. While there are deviations in the force characteristics on the left, especially a time shift at the second force peak, the force characteristics on the right are nearly congruent with the test LT02.

The deformation images in Figure 20 also show that the deformation behaviour in the experiment can be well represented in the simulation. The buckling mode of the crash boxes and the bending mode of the longitudinal member correspond well between the simulation and the test. The deformation images show the longitudinal member system after it has been removed from the trolley.

This means that the residual stress caused by the deformation is relieved after the removal and that the longitudinal members are subject to elastic recovery effects due to an elastic unloading. This is also considered in the simulation by means of a two-step calculation (1. crash event, 2. rebound/settling after disassembling) to allow for an appropriate comparison of the photogrammetric measurement of the specimen with the simulation. The model of the longitudinal member is not as systematically and accurately validated as the model of the crash box; this is the reason for remaining differences in the deformation and force curves.
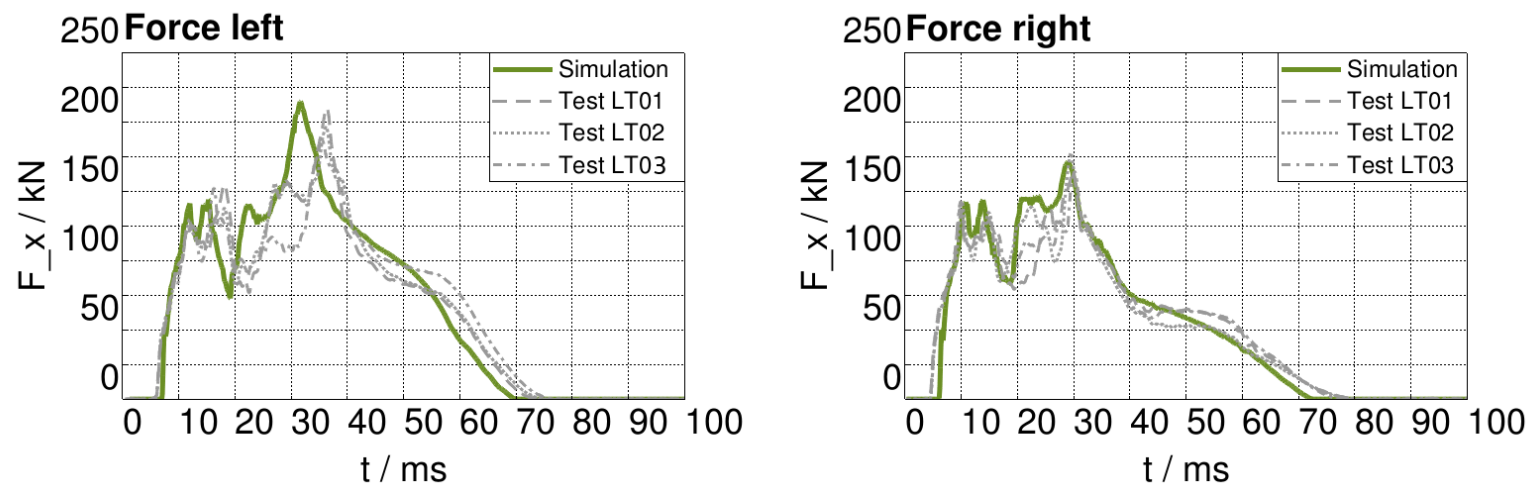

Figure 19: Comparison of force-time curves of simulation and experiment. 


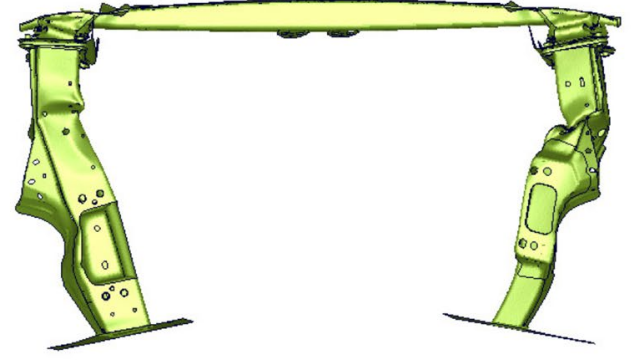

Simulation

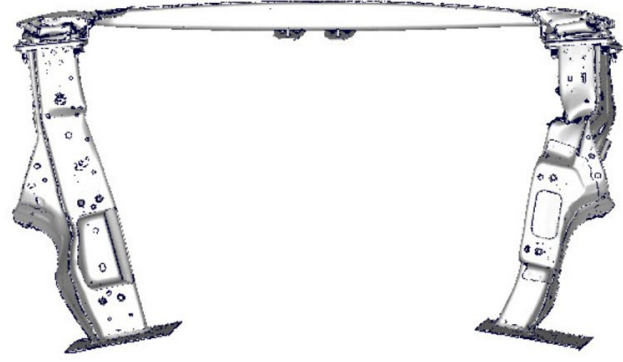

Test

Figure 20: Final deformation modes from simulation and experiment.

This analysis proves that the validated crash box can be well transferred as a sub-model to the longitudinal member system.

\section{Conclusion}

The numerical and experimental study of the crash box showed that the experimental results can be represented in the simulation with high accuracy. The degree of correspondence of the force characteristics and the deformation behaviour increased significantly for the analysed structure by means of the component characterizations which were conducted. This includes the analysis of the punch trajectory, the experiment-specific material characteristics, the geometry, and the thickness. The most important influencing variable was the geometry data obtained from the component measurement. In addition, the study showed that the findings can be transferred well from the slow load case to the fast load case with different load angles. A sufficient discretization (not presented here) is a prerequisite for this.

Due to the assessment of the modified model in the component scenario it could be shown for the analysed load case that the validation of a model with an already validated sub-model can be performed with a high degree of correspondence with regard to the force characteristics and deformation behaviour of the tests. Further studies cover the model validation of longitudinal member systems with different test variations to determine which model parameters are critical for the degree of correspondence.

\section{Acknowledgements}

The author is grateful to Volkswagen AG for making these investigations possible in the first place. Helpful discussions with my colleagues from the CAE methods department of Volkswagen AG are also gratefully acknowledged.

\section{References}

Abedrabbo N, Mayer R, Thompson A, Salisbury C, Worswick M, and van Riemsdijk I. Crash response of advanced high-strength steel tubes: Experiment and model. International Journal of Impact Engineering, 36(8):1044 - 1057, 2009.

Abramowicz W and Wierzbicki T. On the crushing mechanics of thin-walled structures. Journal of Applied Mechanics, 50:724734, 1983.

Alvin KF, Oberkampf WL, Diegert KV, and Rutherford BM. Uncertainty quantification in computational structural dynamics: A new paradigm for model validation. Technical report, Sandia National Laboratories, Albuquerque, NM 87185, 1996.

Belytschko T and Tsay CS. A stabilization procedure for the quadrilateral plate element with onepoint quadrature. International Journal for Numerical Methods in Engineering, 19:pp. 405-419, 1983.

Booker JM. An engineering perspective on UQ for validation, reliability and certification. In Foundations '04 Workshop for Verification, Validation and Accreditation (VV\&A) in the 21st Century, number LA-UR-04-6670, Arizona State University, Tempe, Arizona, 13-15 Oct., 2004.

Carson JS. Convincing users of model's validity is challenging aspect of modeler's job. Industrial Engineering, pages 74-85, 1986. 
Davis PK. Generalizing concepts of Verification, Validation and Accreditation (VV\&A) for military simulation. Technical Report R-4249-ACQ, Santa Monica, CA, 1992.

Eichmueller G and Meywerk M. Stochastic simulation - detailed validation of square tube. NAFEMS Magazine, 2012.

ESI. Virtual Performance Solution - Solver Reference Manual, 2017

Frey HC and Rhodes DS. Theory methodology based upon bootstrap simulation. Quantitative Analysis of Variability and Uncertainty in Environmental Data and Models, 1(Theory Methodology Based Upon Bootstrap Simulation), 1999.

Fyllingen O, Hopperstad OS, and Langseth M. Simulation of top-hat section subjected to axial crushing taking into account material and geometry variations. International Journal of Solids and Structures, pages pp. 6205-6219, 2008a.

Fyllingen O, Hopperstad OS, and Langseth M.Robustness study on the behaviour of top-hat thin-walled high-strength steel sections subjected to axial crushing. International Journal of Impact Engineering, 2008b.

Gottschalk-Mazouz N. Knowledge, Uncertainty and Abduction. 2003.

Hanson MH and Hemez FM. Uncertainty quantification of simulation codes based on experimental data. In 41th AIAA Aerospace Sciences, 2003.

Hemez FM, Booker JM, and Langenbrunner JR. Answering the question of sufficiency: How much uncertainty is enough? In 1st International Conference on Uncertainty in Structural Dynamics, number LA-UR-07-3575, University of Sheffield, UK, 11-13 June, 2007.

Hemez FM. The myth of science-based predictive modelling. In Foundations '04 Workshop for Verification, Validation and Accrediation (VV\&A) in the 21st Century, LA-UR-04-6829, Arizona State University, Tempe, Arizona, 13-15 Oct., 2004.

Hiermaier S. Numerics and material dynamics of crash and impact processes. Fraunhofer Institut für Kurzzeitdynamik, 2003.

Huh $\mathrm{H}$ and Kang WJ. Crash-worthiness assessment of thin-walled structures with the high-strentgh steel sheet. International Journal of Vehicle Design, 30:2-21, 2002.

laccarino G. Introduction to uncertainty quantification. In SIAM Conference on Computational Science and Engineering, Miami, $\mathrm{FL}, 2009$.

Jones N. Material properties for structural impact problems. Advances in Materials and their Applications, $151-163,1993$.

Kokkula S, Langseth M, Hopperstad OS, and Lademo OG. Behaviour of an automotive bumper beam-longitudinal system at 40o_set impact: an experimental and numerical study. Latin American Journal of Solids and Structures, pages pp. 59-73, 2006.

Marczyk J. Computational Stochastic Mechanics in a Meta-Computing Perspective. International Center for Numerical Methods, 1997.

Oscar P and Eduardo RL. Impact performance of advanced high strength steel thin-walled columns. In Proceedings of the World Congress on Engineering 2008 Vol II, July 2-4, 2008, London, UK.

Otubushin A. Detailed validation of a non-linear finite element code using dynamic axial crushing of a square tube. International Journal of Impact Engineering, 21(5):349 - 368, 1998.

Peixinho N, Jones N, and Pinho ACM. Experimental and numerical study in axial crushing of thin walled sections made of highstrength steels. Journal de Physique, 110:717-722, 2003.

Popov, VL. Contact mechanics and friction - from nanotribology to numerical simulation. Springer-Verlag, Berlin, 2009

Reddy S, Abbasi M, and Far M. Multi-cornered thin-walled sheet metal members for enhanced crashworthiness and occupant protection. Thin-Walled Structures, 94:56 - 66, 2015.

Robinson S. Simulation model verification and validation: Increasing the user's confidence. In Proceedings of the 1997 Winter Simulation Conference, Atlanta, Georgia, 7-10 Dec., 1997.

Tarigopula T, Langseth M, Hopperstad OS, and Clausen AH. Axial crushing of thin-walled high-strength steel sections. International Journal of Impact Engineering, 32:847-882, 2006.

Thunert C. CORA User's Manual, 2012.

Trucano TG. Uncertainty quantification at Sandia. Technical Report SAND2000-0524c, Sandia National Laboratories, 2000. 
Wellkamp P, Eichmueller G, and Meywerk M. Validation and uncertainty quantification - a promising approach to improve credibility of crash simulations? In ESI Global Forum, Paris, France, 21-22 May, 2014.

Wojtkiewicz SF, Eldred MS, Fied RV Jr., Urbina A, and Red-Horse JR. Uncertainty quantification in large computational engineering models. Technical Report AIAA-2001-1455, Sandia National Laboratories, Albuquerque, NM 87185 USA, 2001.

Xu F and Wang C. Dynamic axial crashing of tailor-welded blanks (twbs) thin-walled structures with top-hat shaped section. Advances in Engineering Software, 96:70 - 82, 2016.

Xu P, Yang C, Peng Y, Yao S, Zhang D, and Li B. Crash performance and multi-objective optimization of a gradual energyabsorbing structure for subway vehicles. International Journal of Mechanical Sciences, 107:1 - 12, 2016.

Xue L, Lin Z, and Jiang Z. E_ects of initial geometrical imperfection on square tube collapse. Technical report, Shanghai Jiao Tong University, 2013.

Yamashita M, Gotoh M, and Sawairi Y. A numerical simulation of axial crushing of tubular strengthening structures with various hat-shaped cross-sections of various materials. Key Engineering Materials, volume 233 of Key Engineering Materials, pages 193-198. Trans Tech Publications, 122003.

Zhang $\mathrm{X}$ and Zhang $\mathrm{H}$. Crush resistance of square tubes with various thickness configurations. International Journal of Mechanical Sciences, 107:58 - 68, 2016. 